\title{
Detection of anti-dsDNA as a diagnostic tool: a prospective study in 441 non-systemic lupus erythematosus patients with anti-dsDNA antibody (anti-dsDNA)
}

\author{
TOM SWAAK AND RUUD SMEENK
}

From the Central Laboratory of the Netherlands Red Cross Blood Transfusion Service and Laboratory for Experimental and Clinical Immunology of the University of Amsterdam, The Netherlands

SUMMARY The diagnostic significance of anti-double-stranded deoxyribonucleic acid (antidsDNA) determination was evaluated in a prospective manner from 1974 to 1982 in a group of 441 patients without systemic lupus erythematosus whose sera were found to contain antibodies to dsDNA on routine screening (Farr assay). Within one year 69\% (304) of these patients fulfilled the preliminary American Rheumatism Association (ARA) criteria for systemic lupus erythematosus (SLE). Eighty-two of the remaining 137 patients were followed up for several years. At the end of the study $52 \%$ of these patients had also developed systemic lupus erythematosus. Patients who developed systemic lupus erythematosus were characterised by the occurrence of relatively high avidity anti-dsDNA in the circulation compared with patients who did not develop systemic lupus erythematosus. It can be concluded that about $85 \%$ of patients without systemic lupus erythematosus with anti-dsDNA in the circulation will develop SLE within a few years. Taking into account the relative avidity of anti-dsDNA, as determined by calculation of Farr/polyethylene glycol (PEG) ratios, we conclude that patients with relatively high avidity anti-dsDNA are more prone to develop systemic lupus erythematosus than patients with relatively low avidity anti-dsDNA.

Key words: SLE, Farr assay, avidity, prognosis.

Antibodies to deoxyribonucleic acid (DNA) occupy a special position in the study of systemic lupus erythematosus (SLE). The appearance of antibodies to DNA has been alleged to predict exacerbations of disease, ${ }^{12}$ and the diagnostic importance of antidsDNA has recently been stressed by incorporation of its presence in serum as an ARA criterion. ${ }^{34}$

From early studies on DNA binding it was concluded that the Farr assay provides a sensitive and highly specific test for SLE. ${ }^{56}$ On the other hand high values of anti-DNA have also been described in patients with chronic active hepatitis and $Q$ fever endocarditis. ${ }^{7}$ Most of the work on the specificity of

Accepted for publication 30 October 1984.

Correspondence to Dr R Smeenk, Publication Secretariat, Central Laboratory of the Netherlands Red Cross Blood Transfusion Service, PO Box 9406, 1006 AK Amsterdam, The Netherlands.
anti-dsDNA assays has been carried out by testing panels of serum samples from selected patients with well defined clinical disease. In this way we have confirmed the specificity for SLE of anti-dsDNA measured by the Farr assay and by the immunofluorescence test (IFT) on Crithidia luciliae. However, when sera sent to us for routine diagnostic anti-dsDNA determination were tested, the specificity for SLE of both methods was completely lost. $^{8}$ We found antibodies to dsDNA in many patients who were not diagnosed as cases of SLE and did not fulfil the preliminary ARA criteria. The sera we tested came from patients suspected of having SLE, and were therefore negatively selected for actually having SLE; a major reason for sending the sera to us was to obtain further information and to establish a diagnosis. The finding of anti-dsDNA 
in these non-SLE patients could be explained by the hypothesis that non-SLE patients with anti-dsDNA would develop SLE in the future, or that these patients formed a subset of SLE patients.

In order to evaluate the relevance of the presence of anti-dsDNA in non-SLE patients we performed a prospective study, from 1974 until 1982, in which all non-SLE patients with anti-dsDNA in their serum were followed up to see whether they would develop SLE or whether they had an SLE-like syndrome.

\section{Materials and methods}

ANTINUCLEAR ANTIBODY (ANA) DETERMINATION To detect the presence of ANAs in sera of patients the indirect immunofluorescence technique was used. Sera were diluted 1:10 in phosphate-buffered saline (PBS) and incubated for $30 \mathrm{~min}$ at room temperature on acetone-fixed cryostat sections of rat liver tissue. After washing the slides for $30 \mathrm{~min}$ at $30^{\circ} \mathrm{C}$ with PBS (three changes) they were incubated with a 1:60 dilution of a fluorescein isothiocyanate (FITC)-conjugated polyclonal antiserum against human gammaglobulin (lot no. SH 17-01-F05; prepared in this institute). After a final $30 \mathrm{~min}$ washing procedure slides were mounted and read in a Leitz Orthoplan immunofluorescence microscope equipped with incident illumination. Nuclear fluorescence was taken as indication of the presence of ANAs.

ANTI-dsDNA DETERMINATION WITH THE FARR ASSAY Details of the method have already been published. ${ }^{9-12}{ }^{3} \mathrm{H}$-labelled PM2-DNA (100 ng) was incubated with $50 \mu \mathrm{l}$ serum in the presence of $800 \mu \mathrm{g}$ normal human gammaglobulin (HGG). The incubation volume was $0.2 \mathrm{ml}$ and the medium PBS. After incubation of the mixture for one hour at $37^{\circ} \mathrm{C} 5 \mathrm{ml}$ cold $50 \%$ saturated ammonium sulphate was added. The precipitate was allowed to form for $30 \mathrm{~min}$ at $4^{\circ} \mathrm{C}$ and was then centrifuged for 15 minutes at 3000 $g$ in a refrigerated centrifuge, washed twice with $50 \%$ saturated ammonium sulphate, and counted. Mean binding of 135 control sera was $5 \% \pm 3 \%(2 \times$ SD) of the input. Anti-dsDNA activity is expressed in terms of units $/ \mathrm{ml}$, one unit being defined as the amount of anti-dsDNA precipitating $30 \%$ of the added DNA under the above conditions. ${ }^{12}$

ANTI-dsDNA DETERMINATION WITH THE PEG ASSAY Details of this method have already been published. ${ }^{13}{ }^{14}$ To $50 \mu \mathrm{l}$ of serum was added $50 \mu \mathrm{l}$ of a solution of $1.6 \mathrm{~g} / \mathrm{l} \mathrm{HGG}, 50 \mu \mathrm{l}$ of a solution of $0.2 \mathrm{~g} / \mathrm{l}$ dextran sulphate (DXS, Pharmacia Fine Chemicals $\mathrm{AB}$, Sweden, lot no. 5259), $50 \overline{\mu l}$ of a solution of 2 $\mathrm{mg} / \mathrm{l}{ }^{3} \mathrm{H}$-labelled DNA, and $200 \mu \mathrm{l}$ of a solution of $7 \%$ (w/v) polyethylene glycol (PEG 6000, KochLight Laboratories, Colnbrook, UK). All components were dissolved or diluted in PBS. Incubation was carried out for one hour at $37^{\circ} \mathrm{C}$ followed by two hours at $4^{\circ} \mathrm{C}$. After centrifugation for $15 \mathrm{~min}$ at $3000 \times \mathrm{g} 200 \mu \mathrm{l}$ of the supernatant was taken, dissolved in $8 \mathrm{ml} \mathrm{NE}-260 \mathrm{sp}$ (New England Nuclear Corp.), and counted for radioactivity. A $0 \%$ binding control, in which serum was omitted, was included in each experiment. Mean binding of 200 normal control sera was $6 \% \pm 2 \%(2 \times \mathrm{SD})$. The anti-dsDNA activity was expressed in (PEG) units/ml, according to the definition used in the Farr assay.

PATIENTS' SERA

From all patients whose sera were sent to our laboratory in the period from 1974 to 1982 for routine ANA screening those patients found to have ANA (detected by the indirect IFT on rat liver

Table 1 Follow-up data of the anti-dsDNA positive, non-SLE patients

\begin{tabular}{|c|c|c|c|c|c|c|}
\hline \multirow[t]{3}{*}{ Year } & \multirow{3}{*}{$\begin{array}{l}\text { Total number } \\
\text { of subjects }\end{array}$} & \multicolumn{5}{|c|}{ Number of patients followed up } \\
\hline & & \multicolumn{2}{|l|}{ SLE developed } & \multirow{2}{*}{$\begin{array}{l}\text { Non-SLE } \\
\text { patients }\end{array}$} & \multicolumn{2}{|c|}{ Lost during the study } \\
\hline & & Within 1 year & $\begin{array}{l}\text { During the } \\
\text { follow up }\end{array}$ & & Died & Other reasons \\
\hline 1974 & 52 & $42(81 \%)$ & 3 & 1 & - & 6 \\
\hline 1975 & 66 & $48(73 \%)$ & 2 & 5 & 3 & 8 \\
\hline 1976 & 88 & $60(68 \%)$ & 4 & 14 & 2 & 8 \\
\hline 1977 & 61 & $40(66 \%)$ & 2 & 9 & 3 & 7 \\
\hline 1978 & 68 & $47(69 \%)$ & 8 & 7 & - & 6 \\
\hline 1979 & 50 & $29(58 \%)$ & 6 & 4 & 1 & 10 \\
\hline 1980 & 56 & $38(68 \%)$ & 6 & 11 & 1 & - \\
\hline Total & 441 & $304(69 \%)$ & 31 & 51 & 10 & 45 \\
\hline
\end{tabular}

- Mostly caused by transfer to physicians other than those who participated in this study. 
sections) and anti-dsDNA (detected by the Farr assay) were selected for study. Clinical features were obtained by means of a standard questionnaire. Once a year, until the end of our investigation in 1982, an evaluation was made of the course of disease in each patient, with special attention to symptoms incorporated in the preliminary ARA criteria. ${ }^{3}$ If a patient fulfilled at least four of the ARA criteria, the investigation was terminated. Only patients who did not fulfil the ARA criteria were followed up. In this way 441 patients, positive in the Farr assay and negative for the diagnosis of SLE, were studied.

\section{Results}

LONGITUDINAL FOLLOW UP DATA

In the period 1974-82 we studied a total of 441 nonSLE patients whose sera were found to contain antibodies to dsDNA. Within one year of entering the study $58 \%$ (1979) to $81 \%$ (1974) (mean: $69.5 \%$ ) of these patients developed SLE according to the ARA criteria (Table 1). The remaining non-SLE patients $(n=137)$ were studied for varying periods of time, depending on their year of entry into the study. During this follow-up study we lost 55 patients. Ten of these died of non-SLE-related causes and most of the other 45 transferred to physicians who did not participate in this study. The data in Table 2 show that, apart from the group of patients who developed SLE in the first year after entering the study, there was a tendency either to develop SLE in the following three years, or not to do so. If the lost patients $(n=55)$ are considered not to have developed SLE, then $26 \%$ of the patients studied had SLE after five years of follow-up study. If these 55 patients are left out, the conclusion is that $52 \%$ of the patients studied had SLE at that time. The cumulative results are depicted graphically in Fig. 1. A steady increase in the percentage of SLE patients was seen during the first years. However, if a diagnosis of SLE was not reached after five years,

Table 2 Follow-up data of the anti-dsDNA positive, non-SLE patients

\begin{tabular}{|c|c|c|c|}
\hline \multirow[t]{2}{*}{$\begin{array}{l}\text { Follow up } \\
\text { years }\end{array}$} & \multirow{2}{*}{$\begin{array}{l}\text { Number of patients followed } \\
\text { in relation to the duration } \\
\text { of the follow up }\end{array}$} & \multicolumn{2}{|c|}{$\begin{array}{l}\text { Patients who developed } \\
\text { SLE }\end{array}$} \\
\hline & & No. & $\%$ \\
\hline $0-1$ & 386 & 304 & 79 \\
\hline $1-2$ & 82 & 1 & 1 \\
\hline $2-3$ & 81 & 10 & 12 \\
\hline $3-4$ & 60 & 15 & 25 \\
\hline $4-5$ & 41 & 2 & 5 \\
\hline $5-6$ & 32 & 3 & 9 \\
\hline $6-7$ & 20 & - & - \\
\hline $7-8$ & 6 & - & - \\
\hline $8-9$ & 1 & - & - \\
\hline
\end{tabular}

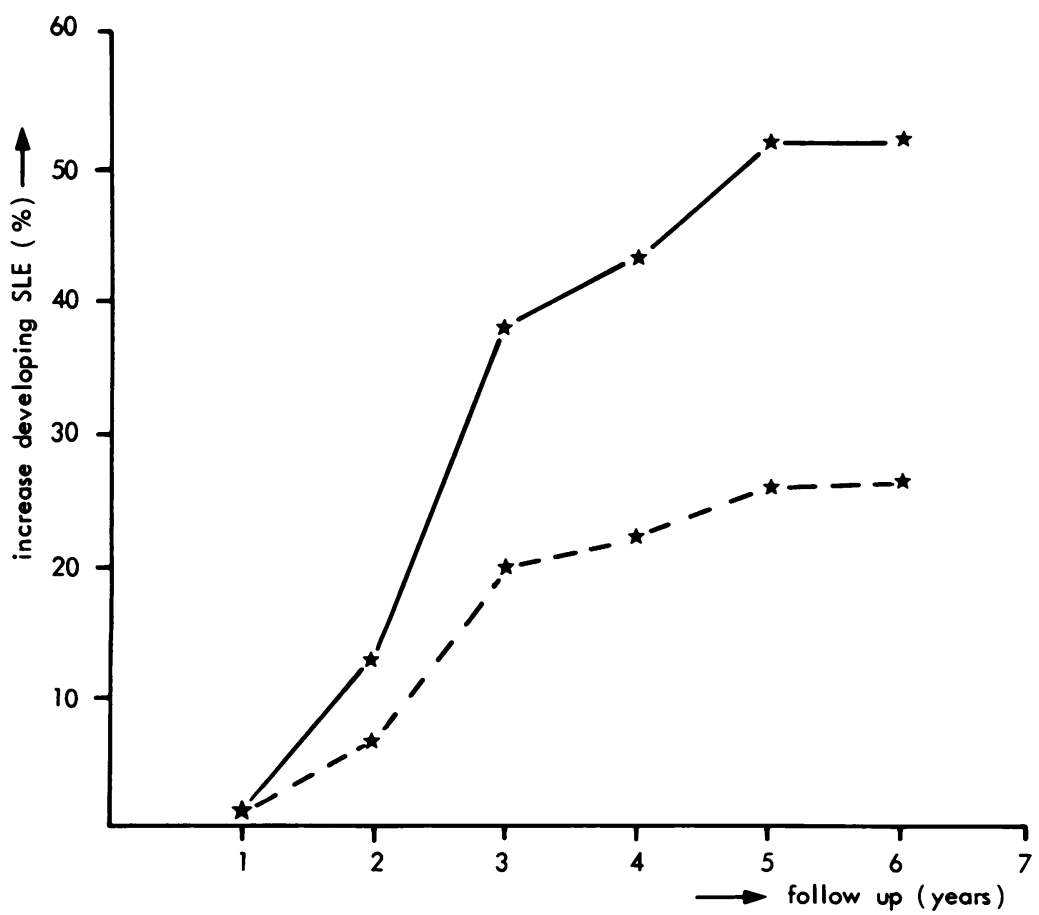

Fig. 1 Cumulative percentage of anti-dsDNA positive, non-SLE patients who developed SLE, expressed as a function of the duration of the follow-up study. *_- ${ }^{*}$ shows the results when the number of patients studied is corrected for patients who died or were lost during the study; *_ _-* shows the results when the number of patients studied is taken as 137. 
then such a patient - albeit having raised titres of anti-dsDNA in the circulation - was not prone to develop SLE. At the end of our study 335 out of the 386 anti-dsDNA positive, non-SLE patients we followed had developed SLE (i.e. $87 \%$ ), mostly during the first year after entering the study (Fig. 2).

AVIDITY IN RELATION TO THE DEVELOPMENT OF SLE Because all sera gave a positive reaction in the Farr assay, they could not be consigned to a low or high avidity anti-dsDNA group on the basis of this test. Expression of the anti-dsDNA content measured with the Farr and PEG assays in units/ml allowed calculation of the ratio between the Farr and PEG results. A high Farr/PEG ratio indicated a relative preponderance of high avidity anti-dsDNA, whereas the opposite held for a low Farr/PEG ratio. ${ }^{15}$ In Fig. 3 the Farr/PEG ratio of patients who developed SLE is compared with the ratio obtained from patients who had still not developed SLE at the end of the study. Patients developing SLE (group I) showed a mean Farr/PEG ratio of 5.4 compared with a Farr/PEG ratio of $2 \cdot 8$ for the non-SLE patients (group II). By drawing a line at a Farr/PEG ratio of

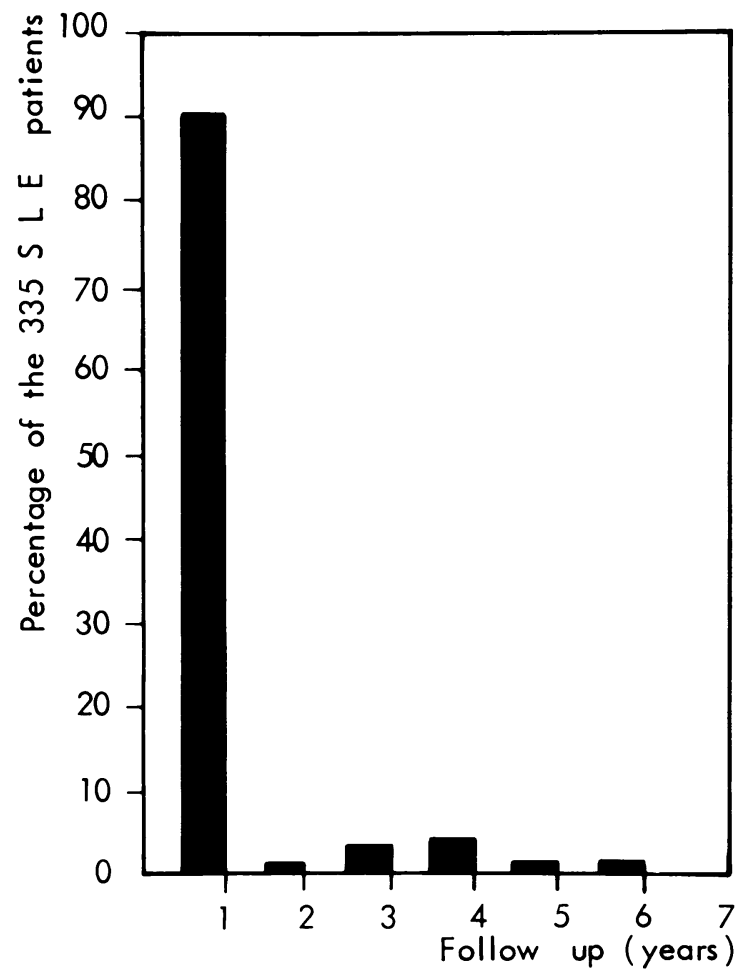

Fig. 2 Percentage of patients who developed SLE $(n=335)$ as a function of the number of years passed since the initial anti-dsDNA detection and the clear development of $S L E$.

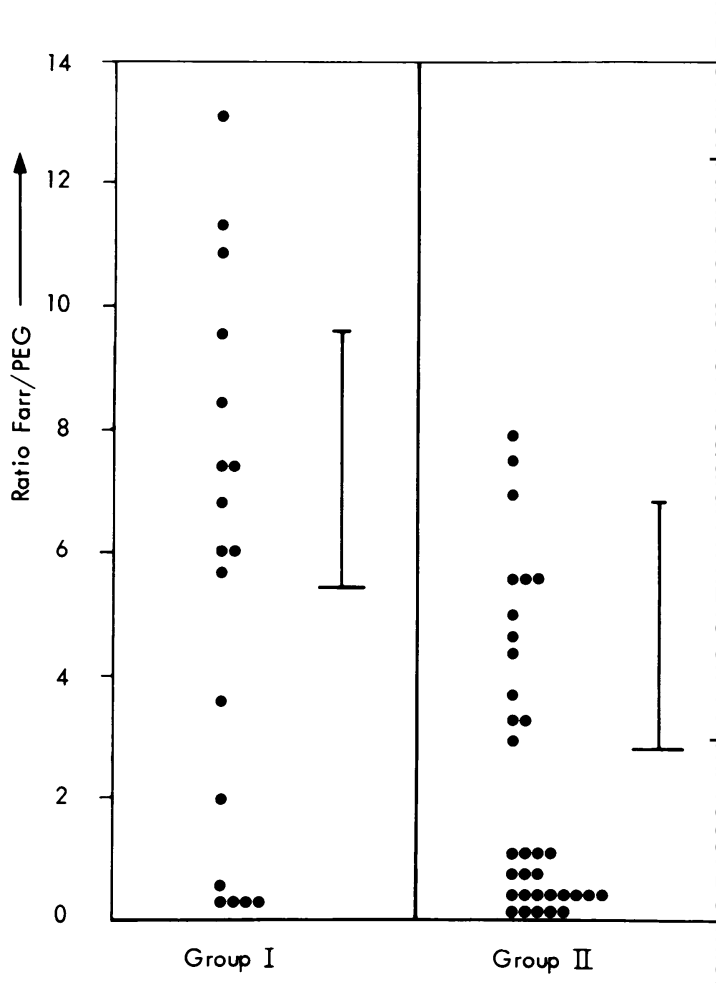

Fig. 3 Relative avidity of anti-dsDNA of two groups of anti-dsDNA positive, non-SLE patients. Group I.

Thirty-one patients who had developed SLE at the end of the study; mean Farr/PEG ratio $=5.4$ (SD 4:0). (Note: In seven cases no serum was available for the PEG assay; in six cases the PEG assay was negative.) Group II. Fifty-on patients who had not developed $S L E$ at the end of the study; mean Farr/PEG ratio $=2 \cdot 8(S D 4 \cdot 0)$. (Note: In 10 cases no serum was available for the PEG assay; in eight cases the PEG assay was negative.)

five, we find that $61 \%$ of the SLE patients (firs? group) have an equal or higher Farr/PEG ration compared with $18 \%$ of the non-SLE patient (second group) $\left(\mathrm{p}<0 \cdot 0901, \chi^{2}=3 \cdot 85\right)$. O

CLINICAL AND LABORATORY FEATURES AT START ON STUDY

Table 3 shows that no clinical differences were detected between patients who developed SLF during the study and those who did not with respecth to the number of preliminary ARA criteria the fulfilled when entering the study. With respect to the actual ARA criteria present a slight differenceD was recorded between the two groups. The inci? dence of ARA criteria 7 and 8 ranged from 68 to $73 \%$ in both groups, but criterion 14 was found in $15 \%$ of the patients in group I and in $30 \%$ of the 
patients in group II. Criteria 10 and 11 were found in only two patients, one in each group.

If Fig. 4 initial anti-dsDNA levels of all patients followed for more than one year are depicted. In

Table 3 Number (percentage) of anti-dsDNA positive, non-SLE patients with a specified number of ARA criteria present at the start of the study

\begin{tabular}{|c|c|c|c|c|}
\hline \multirow[t]{2}{*}{ Group ${ }^{\circ}$} & \multirow{2}{*}{$\begin{array}{l}\text { No. of } \\
\text { patients }\end{array}$} & \multicolumn{3}{|c|}{ Number of positive ARA criteria present } \\
\hline & & 1 & 2 & 3 \\
\hline I & 31 & $5(16 \%)$ & $13(42 \%)$ & $13(42 \%)$ \\
\hline II & 51 & $15(29 \%)$ & $16(31 \%)$ & $20(39 \%)$ \\
\hline
\end{tabular}

Group I consisted of patients who had developed SLE at the end of the study, in contrast with group II patients who had not.

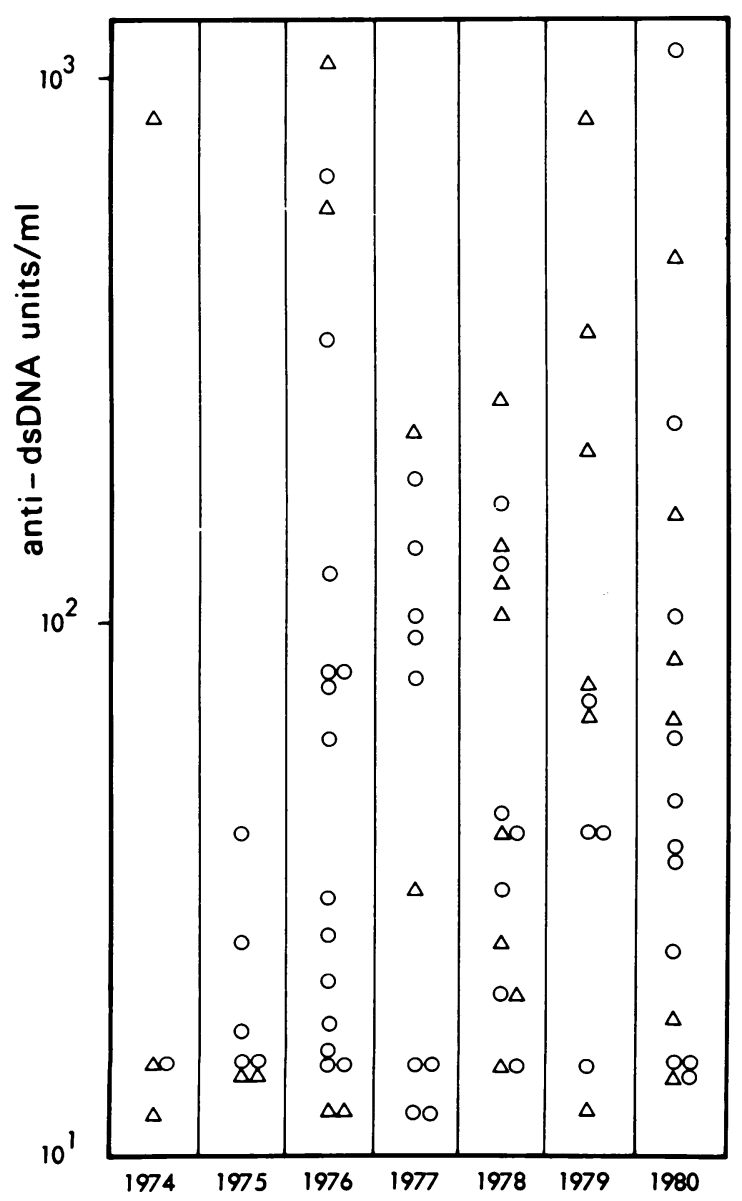

Fig. 4 Initial anti-dsDNA levels of the anti-dsDNA positive, non-SLE patients followed for more than one year. $\mathrm{O}=$ Patients who had not developed $S L E$ at the end of the study. $\triangle=$ Patients who developed SLE during the study. group I a mean anti-dsDNA level of $224 \mathrm{U} / \mathrm{ml}$ (SD 297) was found and in group II a mean value of 124 $\mathrm{U} / \mathrm{ml}$ (SD 300). Statistical calculations did not show any correlation between the development of SLE and initial anti-dsDNA values.

\section{Discussion}

According to the revised ARA criteria for $\mathrm{SLE}^{4}$ the detection of antibodies to dsDNA can support the diagnosis of SLE. Since in the study presented here we sought to establish the relevance of anti-dsDNA determination in the diagnosis of SLE, we used the preliminary ARA criteria to differentiate between SLE and non-SLE in order to exclude anti-dsDNA positivity as a criterion for SLE.

During the period 1974-82 we studied 441 patients with serum antibodies to dsDNA who were not diagnosed as having SLE at the time. Nearly $70 \%$ of these non-SLE patients with anti-dsDNA in the circulation fulfilled the preliminary ARA criteria within one year after they were admitted to the follow-up study. After five years $52 \%$ of the 137 remaining patients had also developed SLE. The figure of $52 \%$ may be an overestimation, since 55 patients were lost during the study. If the presumption is made that all of these patients did not develop SLE, then a figure of $26 \%$ is obtained. On the other hand the possibility that $52 \%$ of these 'lost' patients also developed SLE cannot be excluded. If this were the case, then nearly $80 \%$ of the 137 non-SLE patients would have developed SLE after five years.

With the revised criteria for SLE another 20 patients would be confined to the SLE group at the end of the study. This would mean that over $70 \%$ of the patients who did not have SLE within the first year developed SLE during the five years of follow up study. All our patients were selected on the basis of anti-dsDNA and ANA positivity. As anti-dsDNA and ANAs go hand in hand in these cases, it will - in our view - be an overestimation of the value of these serological findings if both are used as separate criteria for SLE. We think that the diagnosis SLE should be made with caution because of future implications, for example the therapy required and, also the socioeconomic problems which may be raised, e.g., life insurance. With the revised criteria a patient with a quite good prognosis (e.g. mouth ulcers and slight skin rashes combined with a positive ANA test and a positive anti-dsDNA test) may be diagnosed as having SLE.

Our study shows that if anti-dsDNA is detected in a patient (by the Farr assay) the diagnosis SLE according to the preliminary ARA criteria - can in most cases be made within one year. The remaining patients can be divided into two groups: those who 
develop SLE, albeit slowly, and those who after five years still do not have SLE. A question we sought to answer was whether it would be possible to differentiate between antibodies to dsDNA in patients who developed SLE from anti-dsDNA in patients who did not. We have shown before ${ }^{15}$ that the Farr assay only detects DNA antibodies of high avidity, whereas the PEG assay also detects anti-DNA of low avidity. From the results of both assays we could calculate a relative avidity index for each serum. From these data it became clear that patients with high avidity anti-dsDNA developed SLE in a shorter time than patients with low avidity anti-dsDNA. After five years of follow-up study a large proportion of the latter patients had still not developed SLE. There was no significant difference at the start of the study between the number of positive ARA criteria in patients who developed SLE and those who did not. This implies that if a patient is positive for one ARA criterion and has anti-dsDNA in the circulation, the chance for developing SLE is neither greater nor less than if the patient were positive for three ARA criteria. No obvious differences in the clinical features of patients at the start of the study could be found between groups I and II. ARA criteria 7 (arthritis without deformity) and 8 (LE cells) ranged from $68 \%$ to $73 \%$, nearly the same in both groups. A slight difference was found in the frequency of criterion 14 (haemolytic anaemia and/or leucopenia and/or thrombocytopenia); the frequencies in groups I and II were $15 \%$ and $30 \%$ respectively. Criteria 10 (profuse proteinuria $>3.58 \mathrm{mg} / \mathrm{day}$ ) and 11 (cellular casts) were found in only two patients in both groups.

No clear difference between the initially detected anti-dsDNA levels of the two groups was observed (group I: $224 \mathrm{U} / \mathrm{ml}$ (SD 297); group II: $124 \mathrm{U} / \mathrm{ml}$ (SD 300)) as illustrated in Fig. 4. This is in accord with our previous findings ${ }^{17}$ and confirms the absence of a correlation between anti-dsDNA level and disease symptoms or activity. The same study also showed that there is no relation between (initial) anti-dsDNA level and prognosis of disease. It is noteworthy that anti-dsDNA levels of group II patients generally remained stable throughout the follow-up study; in only $20 \%$ of the patients were anti-dsDNA levels decreased or negative at the end of the study. Patients who developed SLE within the first year after initial anti-dsDNA detection could not be distinguished from group I patients either on the basis of the first signs of disease (ARA criteria 7 and 8) or on the basis of sex, age, duration of complaints before the exacerbation, or type of exacerbation. All patients who developed SLE are still being studied, and data on survival rates are being accumulated.
In conclusion we have found that $69 \%$ of a group of 441 non-SLE patients with anti-dsDNA in the $\Rightarrow$ circulation developed SLE within one year. Of the $\stackrel{\text { ? }}{+}$ remaining patients $53 \%$ developed SLE within five years of the follow-up study. Excluding the 55 흘 patients who were lost during follow up, we $\frac{\bar{\sigma}}{\vec{\sigma}}$ followed a total of 386 patients during this prospec- $\propto$ tive study. At the end of the study 331 patients $(87 \%)$ had SLE, which confirms the significance of $\vec{\circ}$ anti-dsDNA determination. We support the incorporation of anti-dsDNA as an ARA criterion but $\vec{\omega}$ would emphasise that the method employed for detection is crucial. Data presented in this papero clearly indicate that patients having high avidity anti-dsDNA in the circulation are more prone to develop SLE than patients with low avidity anti-i dsDNA. The IFT on Crithidia luciliae and the PEG assay also detect anti-dsDNA of low avidity and은 have indeed been found to be less specific for SLEthan the Farr assay which was used throughout this $\frac{D}{0}$ study. ${ }^{7} 16$ Therefore the effective incorporation of anti-dsDNA in the criteria for SLE requires that the $\overrightarrow{0}$ detection method is selective for high avidity anti-co dsDNA. As contamination of the DNA preparation with single-stranded regions also leads to a decrease in specificity of the assay for SLE, ${ }^{9}{ }^{10}$ care should be taken that the antigen preparation used consists of entirely double-stranded DNA. Longitudinal $\stackrel{\circ}{\circ}$ studies of patients having only anti-dsDNA of low avidity are in progress and will be published at a윽 later date.

This study was supported by the Netherlands Leaguc against

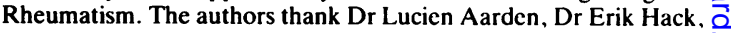
and $\mathrm{Dr}$ Cees Aaij for reading the manuscript critically and Carry $\overline{0}$ Bloklander, Yvonne Kluyt, and Gertrude Damhuis for their help in 3 preparing the typescript.

\section{References}

1 Lightfoot R W, Hughes G R V. Significance of persisting $\frac{D}{2}$ serologic abnormalities in SLE. Arthritis Rheum 1976; 19: 837-43.

2 Swaak A J G, Aarden L A, Statius van Eps L W, Feltkamp T E W. Anti-dsDNA and complement profiles as prognostic $N$ guides in systemic lupus erythematosus. Arthritis Rheum 1979; N 22: 226-35.

3 Cohen S A, Reynolds W F, Franklin E G, et al. Preliminary criteria for the classification of systemic lupus erythematosus. Bull Rheum Dis 1971; 21: 643-8.

4 Tan E M, Cohen A S, Fries J F, et al. The 1982 revised criteria for the classification of systemic lupus erythematosus. Arthritis Rheum 1982; 25: 1271-7.

5 Pincus T, Schur $\mathrm{P}$ H, Rose, J A Decker J L, Talal N. Measurement of serum DNA activity in SLE. $N$ Engl $J$ Med 1971; 281: 701-5.

6 Hughes G R V. Significance of anti-dsDNA antibodies in SLE. Lancet 1971; ii: 861-2.

7 Hughes G R V. Antinucleic acid antibodies in SLE. Clinical and pathological significance. Clin Rheum Dis 1975; 1: 545-59. 
8 Swaak A J G, Groenwold J, Aarden L A, Feltkamp T E W. Detection of anti-dsDNA as a diagnostic tool Ann Rheum Dis 1981; 40: 45-9.

9 Aarden L A. Measurement of anti-dsDNA antibodies. Ann Rheum Dis 1977; 36 (suppl): 91-5.

10 Aarden L A. Lakmaker F, Feltkamp T E W. Immunology of DNA I. The influence of reaction conditions on the Farr assay as used for the detection of anti-dsDNA. J Immunol Methods 1976; 10: 27-37.

11 Aarden L A, Lakmaker F, Feltkamp T E W. Immunology of DNA II. The effect of size and structure of the antigen on the Farr assay. J Immunol Methods 1976; 10: 39-48.

12 Aarden L A, Lakmaker F, Groot E R. Immunology of DNA IV. Quantitative aspects of the Farr assay. J Immunol Methods 1976; 11: 153-63.

13 Riley R L, McGrath J H, Taylor R P. Detection of low avidity
anti-dsDNA antibodies in systemic lupus erythematosus. Arthritis Rheum 1979; 22: 219-25.

14 Smeenk R, Aarden L A. The use of polyethylene glycol precipitation to detect low avidity anti-dsDNA antibodies in systemic lupus erythematosus. J Immunol Methods 1980; 39: $165-80$.

15 Smeenk R, Van der Lelij G, Aarden L. Avidity of antibodies to dsDNA. Comparison of IFT on Crithidia luciliae, Farr assay and PEG assay. $J$ Immunol 1982; 128: 73-8.

16 Smeenk R, Van der Lelij G, Swaak T, Groenwold J, Aarden L A. Specificity to systemic lupus erythematosus of antibodies to dsDNA measured with the polyethylene glycol precipitation assay Arthritis Rheum 1982; 25: 631-8.

17 Swaak A J G, Groenwold J, Aarden L A, Statius van Eps L W, Feltkamp T E W. Prognostic value of anti-dsDNA in SLE. Ann Rheum Dis 1982; 41: 388-95. 